\title{
Clinical, biological and electroencephalographic monitoring of newborns with neurological risk in the Neonatal Intensive Care Unit
}

\author{
FLORINA MARINELA DOANDES ${ }^{1,2}$, ANIKO-MARIA MANEA ${ }^{1,2}$, NICOLETA LUNGU ${ }^{1,2}$, \\ DANIELA CIOBOATA $^{1,2}$, TIMEA BRANDIBUR ${ }^{1,2}$, OANA COSTESCU ${ }^{1,2}$, \\ ANCA HUDISTEANU $^{1}$, EUGEN RADU BOIA ${ }^{3}$ and MARIOARA BOIA ${ }^{1,2}$ \\ ${ }^{1}$ Neonatology and Puericulture Department, 'Victor Babeş' University of Medicine and Pharmacy of Timisoara,
300041 Timisoara; ${ }^{2}$ Neonatology and Preterm Department, 'Louis Țurcanu' Children Emergency Hospital,
300011 Timisoara; ${ }^{3}$ Department of Oto-Rhino-Laryngology, 'Victor Babeş' University of
Medicine and Pharmacy of Timisoara, 300041 Timisoara, Romania
}

Received March 12, 2021; Accepted April 14, 2021

DOI: $10.3892 / \mathrm{etm} .2021 .10192$

\begin{abstract}
Newborns admitted to the Neonatal Intensive Care Unit (NICU) require increased attention regarding neurological assessment and monitoring, due to immaturity or certain conditions that occur during the perinatal and neonatal period. Hypoxic-ischemic encephalopathy (HIE) following perinatal asphyxia is one of the most studied clinical conditions due to the risk of medium- and long-term neurobehavioral outcome. We studied 43 newborns with HIE, for all 3 degrees of impairment, performed amplitude-integrated electroencephalography (aEEG) in the first hours of life and collected common laboratory tests, following serum glycemia at admission and creatinine, creatine kinase (CK) and lactate dehydrogenase (LDH) at admission and in the 3rd day of life. Newborns with mild HIE presented normal aEEG pattern and slightly elevated CK. A total of $80.9 \%$ of the newborns with moderate HIE had seizure patterns in aEEG, while among those with severe HIE, 71.4\% had seizure patterns in aEEG and $28.5 \%$ burst suppression. CK and LDH were mean elevated in those with moderate HIE, and the newborns with severe HIE had also high creatinine values at admission and in the 3rd day of life. Statistically significant differences between the 3 degrees of HIE were noted in terms of creatinine $(\mathrm{P}=0.009)$ and $\mathrm{CK}(\mathrm{P}=0.008)$ at admission and $\mathrm{LDH}$ in the 3rd day of life $(\mathrm{P}=0.036)$. Hypoglycemia was common in our study
\end{abstract}

Correspondence to: Dr Florina Marinela Doandes or Dr Aniko-Maria Manea, Neonatology and Puericulture Department, 'Victor Babeş' University of Medicine and Pharmacy of Timisoara, 2 Eftimie Murgu Square, 300041 Timisoara, Romania

E-mail: florina_doandes@yahoo.com

E-mail: aniko180798@yahoo.com

Key words: hypoxic-ischemic encephalopathy, neonatal seizures, amplitude-integrated electroencephalography, creatinine, lactate dehydrogenase, creatine kinase group. In conclusion, common blood tests in association with aEEG monitoring and rigorous neurological assessment can predict short-term outcome of HIE and multiorgan dysfunction and can help clinicians predict even long-term outcomes in severe HIE.

\section{Introduction}

Due to immaturity or certain conditions that occur during the perinatal and neonatal period, newborns from the Neonatal Intensive Care Unit (NICU) have a high risk of neurological and developmental sequelae. The fetal brain is vulnerable to severe and sustained hypoxia during birth, which can lead to hypoxic-ischemic encephalopathy (HIE) (1). The incidence of perinatal asphyxia is reported to be between 1 and 6 per 1,000 live full-term births (2) and it has been determined to be the third most common cause of neonatal death $(23 \%)$, after preterm birth (28\%) and severe infections (26\%) $(3,4)$. HIE, following perinatal asphyxia, remains an important problem, in both full-term and near-term newborns (5). Early diagnosis and proper treatment of neurological problems in neonatal period and especially of neonatal seizures can reduce adverse neurological outcome in early infancy $(1,6,7)$.

HIE is a term to define the disturbed neurological function in the earliest days of life in the term newborn, consisting in the alteration of the level of consciousness, tone and neonatal reflexes, including the sucking and swallowing reflex, the presence of seizures in moderate and severe stages of HIE $(5,8)$ and cardiovascular (bradycardia, hypotension) and respiratory disorders (irregular respiration, apnea) in severe HIE $(5,9)$. Furthermore, severe HIE includes multisystem involvement, not only neurological, but also cardiac, renal, liver damage $(4,5,10-12)$ and fluctuations in the glycemic values (generally, hypoglycemia) (4).

For the detection of cerebral insult secondary to perinatal hypoxia, electroencephalographic monitoring (EEG) is used, both to detect and monitor seizure activity and also to define abnormal patterns such as 'burst suppression' pattern 
found in severe stages of HIE, low voltage or flat, isoelectric trace (13). Neonatal seizures are often subclinical and difficult to diagnose, and the initiation of anticonvulsant therapy may be delayed to the detriment of the neurological status of the newborn (14). Sometimes some abnormal movements of the newborn (jitteriness) can be interpreted as seizures and, thus, treated incorrectly (15). Therefore, in NICU departments, amplitude-integrated electroencephalography (aEEG) is valuable. The aEEG bedside monitoring utilizes a reduced number of electrodes that are maintained for at least $24 \mathrm{~h}$ (13-15).

The hypoxic-ischemic insult is usually systemic, not only the brain being affected. Severity of HIE may vary, and concurrent multiorgan dysfunctions are commonly observed $(4,11,16,17)$. The aim of this study was to assess newborns with HIE clinically, biologically and electroencephalographically following perinatal asphyxia in the NICU, knowing that the severity of the disease reflects the medium- and long-term outcome of the patients. Neurological recovery is fast in the mild form, while stages 2 and 3 of HIE require long-term medical support, depending on the degree of organ compromise $(9,13)$.

\section{Patients and methods}

Newborns. This single-center prospective study performed over a period of 2 years included newborns with HIE admitted to the regional level III Neonatal Intensive Care Unit (NICU) of 'Louis Turcanu' Emergency Clinical Hospital for Children in Timisoara, Romania.

The inclusion criteria were: Gestational age (GA) $\geq 36$ weeks, clinical and biological history of perinatal asphyxia, not more than $48 \mathrm{~h}$ of life at admission, clinical criteria of mild, moderate or severe HIE, aEEG monitoring for at least $24 \mathrm{~h}$, in the first $72 \mathrm{~h}$ of life.

Exclusion criteria were: GA $<36$ weeks, inability to perform aEEG in the first $72 \mathrm{~h}$ of life, acute meningitis or encephalitis, other severe infections (sepsis or pneumonia), neonatal encephalopathy of other causes than perinatal asphyxia, severe malformations.

The study was approved by Ethics Committee for Scientific Research of the Emergency Hospital for Children 'Louis Turcanu' (approval no. 78/2020). The authors ensure that the study was carried out in accordance with the Declaration of Helsinki.

Demographic variables and clinical data were collected (sex, gestational age, Apgar scores, neonatal resuscitation, mechanical ventilation, neonatal seizures), aEEG, and usual laboratory tests [glycemia, creatinine, creatine kinase, lactate dehydrogenase (LDH)].

Determination of HIE. All newborns were neurologically examined daily and were classified with mild, moderate or severe HIE. We considered normal or neurologically unaffected newborns with an alert level of consciousness, normal spontaneous activity, normal posture, tone and suck reflex, strong Moro reflex, equal pupils and reaction to light, normal heart rate and respiration. We used the modified clinical Sarnat score to grade HIE (9). Mild encephalopathy (stage 1) was considered in hyperalert newborns, with normal/increased spontaneous activity and tone, normal/incomplete suck reflex, strong Moro reflex, low threshold. Moderate encephalopathy (stage 2) was defined in the case of lethargic newborns, with decreased activity, hypotonia, distal flexion and complete extension, weak sucking reflex, incomplete Moro reflex, miosis, bradycardia, and periods of apnea. Severe encephalopathy (stage 3 ) has been associated with stupor or coma, no spontaneous activity, intermittent decerebration posture, flaccidity, absent Moro and suck reflex, pupils deviated/dilated, poor reaction to light, variable heart rate and apnea.

aEEG monitoring. The newborns were aEEG monitored in the NICU using Natus Cerebral Function Monitor- Olympic Brainz Monitor. Cross cerebral 1 or 2 channel monitoring was used ( 3 or 5 electrodes), with linear display of low-voltage activity $(0-10 \mu \mathrm{V})$ and logarithmic display of activity between $10-100 \mu \mathrm{V}$, aEEG displayed as a time-compressed trend at $6 \mathrm{~cm} / \mathrm{h}$. The aEEG was classified as: a) Continuous normal voltage (CNV) and discontinuous normal voltage (DNV) without abnormal changes: normal; b) burst suppression (BS) and inactive flat trace (FT): Abnormal; c) single or repeated seizures $(\mathrm{Sz})$ and status epilepticus (SE): epileptic activity $(18,19)$.

Laboratory tests. Laboratory common tests were processed in our hospital laboratory upon admission to the NICU. In our study, we considered serum creatinine, creatine kinase and LDH nonspecific markers of kidney and brain damage following perinatal hypoxia. We also evaluated serum glycemia at admission. Analyses in the 3rd day of life were also collected. Normal values (from hospital laboratory devices) consisted of: Glycemia, 3.88-6.38 mmol/l; creatinine, 21-75 $\mu \mathrm{mol} / 1$; LDH, 225-600 U/l; and total creatine kinase, 24-228 U/1. If several determinations of the same analysis were performed, the highest or lowest value was recorded as significant, depending on the case.

Statistical analysis. Statistical analyses were performed using Epi Info 7 for Windows (https://www.cdc.gov/epiinfo/pc.html). We used ANOVA test to analyze the variation of laboratory tests according to HIE stage. If ANOVA test could not be applied, we used Mann-Whitney or Kruskal-Wallis test. A P-value of $<0.05$ was considered indicative of a statistically significant difference.

\section{Results}

Assessment of HIE. Forty-three newborns with gestational age $\geq 36$ weeks and HIE were enrolled in the study. Of these, $15(34.9 \%)$ had mild HIE, 21 (48.8\%) moderate HIE and $7(16.2 \%)$ had severe HIE (Table I), according to the modified clinical Sarnat score (9).

Girls were predominant in the group of newborns with mild HIE (60\%), while boys predominated in the group of newborns with moderate (76.2\%) and severe (57.1\%) HIE.

All newborns with mild HIE had an Apgar score over 6 at $5 \mathrm{~min}$, and a small percentage $(13.3 \%)$ required resuscitation maneuvers at birth. However, of this group, 20\% developed clinically observed neonatal seizures in the first days of life, and in $13.3 \%$ of these, aEEG records confirmed the seizures. They began anti-seizure treatment and were subsequently classified as stage 2 of HIE, with biological tests at admission 
Table I. Characteristics of the newborns with HIE ( $\mathrm{N}=43)$, according to the degree of neurological impairment.

\begin{tabular}{|c|c|c|c|c|}
\hline & Mild HIE (n=15) & Moderate HIE (n=21) & Severe HIE $(n=7)$ & P-value \\
\hline \multicolumn{5}{|l|}{ Sex, n $(\%)$} \\
\hline Girls & $9(60 \%)$ & $5(23.8 \%)$ & $3(42.8 \%)$ & \\
\hline Boys & $6(40 \%)$ & $16(76.2 \%)$ & $4(57.1 \%)$ & \\
\hline \multicolumn{5}{|l|}{ Apgar score at $5 \min , \mathrm{n}(\%)$} \\
\hline $0-5$ & $0 \quad(0 \%)$ & $4(19.0 \%)$ & $5(71.4 \%)$ & \\
\hline $6-10$ & $15(100 \%)$ & $17(80.9 \%)$ & $2(28.5 \%)$ & \\
\hline Neonatal resuscitation (delivery room), n (\%) & $2(13.3 \%)$ & $11(52.4 \%)$ & $7(100 \%)$ & \\
\hline Mechanical ventilation, n (\%) & 0 & $10(47.6 \%)$ & $7(100 \%)$ & \\
\hline Symptomatic seizures, n (\%) & $3(20 \%)$ & $17(80.9 \%)$ & $3(42.8 \%)$ & \\
\hline \multicolumn{5}{|l|}{ aEEG, n (\%) } \\
\hline Normal (CNV, DNV) & $13(86.6 \%)$ & $4(19.0 \%)$ & 0 & \\
\hline Seizures/SE & $2(13.3 \%)$ & $17(80.9 \%)$ & $5(71.4 \%)$ & \\
\hline Abnormal (BS, FT) & 0 & 0 & $2(28.5 \%)$ & \\
\hline \multicolumn{5}{|l|}{ Laboratory evaluation } \\
\hline \multicolumn{5}{|l|}{ At admission (First $48 \mathrm{~h}$ of life) (mean values) } \\
\hline Glycemia $(\mathrm{mmol} / \mathrm{l})(\mathrm{NV}=3.88-6.38)$ & 3.34 & 3.57 & 2.95 & 0.429 \\
\hline Creatinine $(\mu \mathrm{mol} / \mathrm{l})(\mathrm{NV}=21-75)$ & 37.42 & 63.33 & 85.85 & $0.009^{\mathrm{a}}$ \\
\hline $\mathrm{LDH}(\mathrm{U} / \mathrm{l})(\mathrm{NV}=225-600)$ & 592.00 & 935.55 & $1,319.00$ & 0.052 \\
\hline $\mathrm{CK}$ total $(\mathrm{U} / \mathrm{l})(\mathrm{NV}=24-228)$ & 325.28 & $1,017.33$ & $1,332.00$ & $0.008^{\mathrm{a}}$ \\
\hline \multicolumn{5}{|l|}{$72 \mathrm{~h}$ of life (mean values; same values as normal) } \\
\hline Creatinine $(\mu \mathrm{mol} / \mathrm{l})$ & 41.71 & 58.88 & 99.57 & 0.664 \\
\hline $\mathrm{LDH}(\mathrm{U} / \mathrm{l})$ & 418.42 & 629.22 & 904.14 & $0.036^{\mathrm{a}}$ \\
\hline CK total (U/l) & 285.57 & 465.66 & 831.00 & 0.066 \\
\hline
\end{tabular}

${ }^{a} \mathrm{P}<0.05$. HIE, hypoxic-ischemic encephalopathy; aEEG, amplitude-integrated electroencephalography; CNV, continuous normal voltage; DNV, discontinuous normal voltage; SE, status epilepticus; BS, burst suppression, FT, flat trace; CK, creatine kinase (average value); LDH, lactate dehydrogenase (average value); NV, normal values.

indicating also evidence of mild multisystem involvement. All patients with severe HIE required resuscitation maneuvers in the delivery room and non-invasive $(42.8 \%)$ or invasive $(57.1 \%)$ respiratory support in the NICU. The Apgar score at $5 \mathrm{~min}$ was not higher than 5 for $71.4 \%$ of this group.

aEEG monitoring. The aEEG monitoring of the newborns was performed for a minimum of $24 \mathrm{~h}$, as soon as possible after hospitalization, for all the patients with a history of perinatal asphyxia, immediately after clinical and neurological evaluation, but not later than $48 \mathrm{~h}$ of life. Newborns with mild HIE had normal background pattern. In the group of newborns with moderate HIE, only $19 \%$ had a normal course during aEEG recordings. They did not require mechanical ventilation, but they had disorders of tone, sucking and swallowing and had a favorable evolution. The other $80.9 \%$ had manifested clinical seizures, which were found entirely in the aEEG trace. They required assisted mechanical ventilation up to $58.8 \%$ and continuous medical care in the intensive care unit, for 10 days on average. Out of those with severe HIE, $71.4 \%$ had electrographic seizures or status epilepticus during aEEG monitoring, although only $42.8 \%$ of them presented clinical manifestations. Two patients with severe HIE (28.5\%) had a 'burst suppression' (BS) trace. During aEEG monitoring, one continued with BS trace and the other evolved towards flat trace (FT), both requiring cardio-respiratory support in the NICU.

Laboratory test results. Upon admission of the patients in the NICU, basic blood tests were performed. Analyses in the 3rd day of life were also performed. Most of the newborns with neonatal hypoxic encephalopathy experienced hypoglycemia upon admission, regardless of the degree of neurological impairment or the presence or absence of neonatal seizures (Table I). Patients with mild encephalopathy had slightly elevated serum creatine kinase levels, both at admission and in the 3rd day of life. Newborns with moderate encephalopathy had, on average, LDH and creatine kinase with elevated values, while infants with severe HIE had elevated mean values of all three biological markers. Statistically significant differences were observed between the 3 groups of patients with different degrees of perinatal HIE in terms of laboratory tests. Serum creatinine and serum creatine kinase values at admission were significantly higher in the group of patients with severe or moderate HIE ( $\mathrm{P}=0.009$ and $\mathrm{P}=0.008$, respectively). From the analyses performed at $72 \mathrm{~h}$ of life, statistically significant 
differences between the 3 categories of encephalopathy were noted only in the case of $\mathrm{LDH}(\mathrm{P}=0.036)$.

\section{Discussion}

Neonatal HIE is still an important and common condition in NICUs, and moderate and severe stages should be closely assessed due to long-term neurological morbidity $(4,20,21)$. The clinical classification of newborns in one of the 3 degrees of HIE is useful for the short-term prognosis of the newborn, but also for medium and long-term outcome. The electroencephalographic recording (22) and biological tests of multisystemic involvement are also valuable in the outcome assessment $(4,23,24)$.

aEEG has become a standard device for assessments of the neonates in the NICU, especially full-term infants with perinatal asphyxia and neonatal HIE (25). The combination of early aEEG and clinical examination within the first $12 \mathrm{~h}$ of birth increased the positive predictive value and specificity compared with either method alone $(26,27)$. Moreover, aEEG is a reliable device for an accurate diagnosis of neonatal seizures and monitoring the response of anti-seizure medication $(25,28)$. Changes in the electroencephalographic background pattern are a predictor of long-term outcome $(25,29)$. A normal EEG pattern is correlated with a good, normal outcome, while 'burst suppression' is a negative prognostic factor, even death (30).

In the present study, the newborns with mild HIE had normal/quasi-normal background pattern: Continuous normal voltage or discontinuous normal voltage, except $13.3 \%$ who had seizures, both clinically and electroencephalographically. Clinical neonatal seizures in the setting of birth asphyxia are associated with worse neurodevelopmental outcome, independently of the severity of hypoxic-ischemic brain injury (31). Neonatal seizures in the context of HIE change the staging of neurological impairment. In the present study, newborns with moderate hypoxic encephalopathy who had a normal aEEG pattern (19\%) exhibited a good neurological outcome, while those with a seizure pattern required longer care in the NICU and mechanical ventilation (58.8\%). Among the newborns with severe HIE, 71.4\% had clinical seizures, correlated with a typical pattern found in aEEG records, and in $28.5 \%$ the aEEG route was 'burst suppression' (BS). The evolution was towards death in $14.2 \%$.

For the treatment of severe HIE, efforts have been made in order to identify specific neuroprotective therapies able to block or reduce the negative effects of hypoxia and ischemia (32). Hypothermia represents the treatment of choice for term newborns with HIE following perinatal asphyxia and the only therapy having neuroprotective effects in larger clinical studies (4). Before and during therapeutic hypothermia, the newborns are electroencephalographic assessed (aEEG) to monitor the outcome of the procedure. Our NICU does not own a device for therapeutic hypothermia. This procedure must start during the first $6 \mathrm{~h}$ of life (32). The newborns admitted to our intensive care unit are transferred from a level 1 or 2 maternity and the transfer procedures usually exceed the first $6 \mathrm{~h}$ of life. In our NICU, aEEG monitoring is useful to accurately classify newborns at the appropriate stage of neurological injury, providing us valuable information about brain injury severity, and this monitoring also guides us in the continuous monitoring of anti-seizure medication in patients with neonatal seizures and in the prognosis for subsequent neurobehavioral outcomes.

EEG monitoring in the NICU may assist the neonatologist in the prediction of medium- and long-term outcome of neurological impairment, subsequent motor, mental, and cognitive deficiencies, and the quality of life of survivors (33). Newborns with severe intrapartum hypoxia, after cardio-respiratory stabilization, may develop severe brain damage and are extremely difficult to care for at home. The communication of the outcome to the parents of these children is important, and a flat/inactive EEG trace may help us to predict the neuronal destruction, and the long-term outcome (34). A retrospective study (35) indicated that recovery of the background pattern to normal is not uncommon, and that the sooner the background pattern normalizes, the better the prognosis. Similarly, infants with severely abnormal patterns at $6 \mathrm{~h}$, that recover to normal by $24 \mathrm{~h}$, exhibit mild or no disability in $61 \%$ of infants (36). Two different recent studies that examined the relationship between electrographic seizures and long-term outcome in neonates with HIE, concluded that the presence of seizures per se was not associated with abnormal outcome. However, a high seizure burden as well as a persistent abnormal aEEG background pattern are significantly associated with a poor prognosis $(22,37)$.

Clinical detection of neonatal seizures is difficult, because not all the infants have clinical manifestations and their detection represents a particular diagnostic challenge in the NICU $(38,39)$. Numerous studies have indicated that about $80 \%$ of EEG seizures in neonates have no associated clinical symptoms, and therefore would not be identified without continuous EEG monitoring even by expert clinicians. Moreover, there are no differences in the degree of encephalopathy and the electroclinical seizures or EEG-only seizures $(7,40)$.

In our study, newborns with second degree of encephalopathy that had seizure pattern in aEEG, also manifested clinical seizures, observed by the medical staff, and most of them were subtle (chewing, pedaling or bicycling movements, ocular movements as blinking or nystagmus, apnea or excessive salivation). Newborns with 3rd degree HIE, $42.85 \%$ presented correlation between EEG and clinical manifestation and $28.6 \%$ only had a seizure pattern on EEG. Neonatal seizure follow-up studies concluded that 3rd stage Sarnat HIE and an abnormal neonatal EEG pattern are associated with poor neurological outcome (41).

Imaging techniques, such as cranial ultrasound scans and magnetic resonance imaging (MRI), are useful for prognosis of newborns with HIE, but not until 24 h or more after birth (42). EEG is extremely useful for the first $24 \mathrm{~h}$ (19).

The Apgar score is a clinical indicator that reflects the newborn's physical condition at birth. Perinatal asphyxia, but also other risk factors, such as severe infections, prematurity, maternal analgesia may cause a low Apgar score and the need for cardiopulmonary resuscitation at birth $(4,43)$. Most newborns respond rapidly to resuscitation maneuvers and their outcome is very good, but in the case of newborns that do not respond to resuscitation in the first $10 \mathrm{~min}$ of life, the probability of surviving with severe disabilities is high (4). 
Antioxidant defense is deficient in newborns and can be enhanced by the action of reactive oxygen species, generated by perinatal diseases, such as neonatal respiratory distress or birth asphyxia. Studies on relatively large numbers of term newborns with asphyxia have demonstrated the importance of resuscitation with atmospheric air in limiting injury and improving the survival rate (44-46). Apgar scores provide useful prognostic data before other evaluations are available for infants with HIE. Death or moderate/severe disability is common but not uniform with Apgar scores of <3 (47).

In our study, $79.1 \%$ of the patients had an Apgar score at 5 min between 6 and 10; 50\% of them were classified as HIE stage 2 and $5.9 \%$ presented severe HIE upon clinical examination in our department. All patients with mild HIE had an Apgar score at 5 min of $\geq 6$. Among patients with Apgar score at $5 \mathrm{~min} \leq 5,55.5 \%$ had severe neonatal encephalopathy according to Sarnat staging, and $44.4 \%$ moderate encephalopathy. We can also conclude from our study that a 5-min Apgar score $<5$ can be associated with moderate and severe HIE. Low Apgar score is common for moderate and severe HIE, but is a nonspecific finding (4).

Literature data suggest that no single biomarker is able to assess neurological damage after perinatal asphyxia, but a panel of multiple biomarkers should be considered in evaluating the neurological outcome of asphyxia, the clinical consequences and the initiating or stopping of neuroprotective therapy (4). Hypoxia-ischemia causes a direct damage to the myocardium which, together with the negative consequences of compensatory mechanisms to maintain cerebral perfusion, leads to a recognizable clinical and laboratory picture. Kidney injury represents the best systemic marker of brain injury. Fluctuation of blood glucose concentration may be observed, hypoglycemia being the most common $(4,16,48)$. LDH is a marker of cellular damage as a result of hypoxia-ischemia in affected organs (49). In neonatal HIE patients with a poor prognosis, significantly higher levels of LDH and serum creatine kinase $(\mathrm{CK})$ are observed compared with neonatal HIE patients with a good prognosis $(17,21,50)$. The sensitivity and specificity of LDH alone for the diagnosis of HIE are lower than when serum CK, LDH, uric acid and lactic acid are simultaneously determined (specificity and sensitivity are 87 and 94\%, respectively) (23). A recent study (11) showed a positive correlation between multiorgan disfunction, HIE severity and infant death. Also, the probability of mortality is higher in stage $3 \mathrm{HIE}$ infants with observed multiorgan dysfunctions (11). There are also differences in the number of affected organs on day 1 and during the first 3 days of life, according to HIE stage, and in absence of multiple organ dysfunction, a perinatal hypoxic-ischemic etiology of acute severe neonatal encephalopathy should be carefully reconsidered (51)

In our study, admission hypoglycemia was present in most newborns. Creatinine, $\mathrm{CK}$ and LDH had elevated mean values in the neonates with severe HIE, both at admission and in the 3rd day of life. In patients with moderate HIE, only LDH and CK were elevated, and newborns with mild HIE showed slightly elevated CK. Statistically significant differences between the 3 groups of our study were noted in terms of creatinine $(\mathrm{P}=0.009)$ and $\mathrm{CK}(\mathrm{P}=0.008)$ at admission and $\mathrm{LDH}$ in the 3 rd day of life $(\mathrm{P}=0.036)$. In addition, future research should optimize the use of multiple biomarkers to establish a unified, standard method to improve the accuracy of neonatal HIE diagnosis (50).

Specific analyses for perinatal asphyxia studied to date can be expensive and the cost/efficiency ratio must be weighed. Common biochemical tests, along with bedside electroencephalographic recording, can estimate the current neurological and systemic impairment and can also be predictive of outcome. Postnatal evaluation after perinatal asphyxia using aEEG can reliably predict neurodevelopmental outcome, as early as $3 \mathrm{~h}$ after birth (19).

Neonatal HIE remains a leading cause of long-term neurological morbidity and significant cause of mortality $(4,34,48)$, despite innovative postpartum treatments $(29,51)$ and early recognition of fetal neurological impairment (52).

aEEG is a simple, non-invasive method of assessing cerebral electric activity; it has a predictive role in neurological outcome and it detects abnormal brain activity, which cannot be detected by imaging methods. Common laboratory tests can guide us in assessing the degree of systemic damage in HIE secondary to perinatal asphyxia, in conjunction with a competent neurological assessment and continuous bedside electroencephalographic monitoring.

Limitations of the study. Limitations of the study include a lack of neurological follow-up after discharging the infants from our hospital ward. It was not possible to study serum creatine kinase muscle-brain specific fraction (CK-BB) in our hospital laboratory, but only total CK.

\section{Acknowledgements}

Professional editing, linguistic and technical assistance was performed by Irina Radu.

\section{Funding}

No funding was received.

\section{Availability of data and materials}

The datasets used and/or analyzed during the current study are available from the corresponding author upon reasonable request.

\section{Authors' contributions}

FMD was responsible for conception and design, analysis and interpretation of data, and drafting of the manuscript. AMM was responsible for conception and design, and drafting of the manuscript. NL was responsible for conception and design, and drafting the manuscript. DC was responsible for acquisition of data, analysis and interpretation of data. TB was responsible for drafting the manuscript, conception and design. OC and ERB were responsible for the design of the study and drafting the manuscript. AH was responsible for acquisition of data, conception and design. $\mathrm{MB}$ was responsible for analysis and interpretation of data, coordination, revision and final approval of the version to be published. All authors read and approved the final manuscript. 


\section{Ethics approval and consent to participate}

Ethics approval was obtained from the Ethics Committee for Scientific Research of the 'Louis Turcanu' Emergency Hospital for Children in Timisoara, Romania (approval no. 78/2020). Parental or caregiver consent was obtained where applicable and was attached to the patient's medical record document. This publication and database do not contain personal data, do not compromise anonymity or confidentiality or breach local data protection laws.

\section{Patient consent for publication}

Not applicable.

\section{Competing interests}

The authors declare that they have no competing interests.

\section{Authors' information}

First author Florina Marinela Doandes is a $\mathrm{PhD}$ student at 'Victor Babeș' University of Medicine and Pharmacy of Timisoara, 300041 Timisoara, Romania.

\section{References}

1. Graham EM, Ruis KA, Hartman AL, Northington FJ and Fox HE: A systematic review of the role of intrapartum hypoxia-ischemia in the causation of neonatal encephalopathy. Am J Obstret Gynecol 199: 587-595, 2008.

2. de Haan M, Wyatt JS, Roth S, Vargha-Khadem F, Gadian D and Mishkin M: Brain and congnitive-behavioural development after asphyxia at term birth. Dev Sci 9: 350-358, 2006.

3. Lawn JE, Cousens S, Zupan J and for the Lancet Survival Steering team: 4 million neonatal deaths: When? Where? Why? Lancet 365: 891-900, 2005.

4. Antonucci R, Porcella A and Pilloni MD: Perinatal asphyxia in the term newborn. J Pediatr Neonat Individual Med 3: e030269, 2014.

5. Robertson NJ and Groenendaal F: Hypoxic-ischaemic brain injury. In: Rennie and Robertson's Textbook of Neonatology. 5th edition, 40.4:. Elsevier Limited, pp114-1155, 2012.

6. Zaigham M, Lundberg F and Olofsson P: Protein S100B in umbilical cord blood as a potential biomarker of hypoxic-ischemic encephalopathy in asphyxiated newborns. Early Hum Dev 112: 48-53, 2017.

7. Massey SL, Jensen FE and Abend NS: Electroencephalographic monitoring for seizure identification and prognosis in term neonates. Semin Fetal Neonatal Med 23: 168-74, 2018.

8. Nelson K and Leviton A: How much of neonatal encephalopathy is due to birth asphyxia? Am J Dis Child 145: 1325-1331, 1991

9. Sarnat HB and Sarnat MS: Neonatal encephalopathy following fetal distress: A clinical and electroencephalographic study. Arch Neurol 33: 696-705, 1976 .

10. Elsadek AE, FathyBarseem N, Suliman HA, Elshorbagy HH, Kamal NM, Talaat IM, Al-Shokary AH, Abdel Maksoud YH, Ibrahim AO, Attia AM, et al: Hepatic injury in neonates with perinatal asphyxia. Global Pediatric Health 8, 2021.

11. Michniewicz B, Al Saad SR, Karbowski LM, Gadzinowski J, Szymankiewicz M and Szpecht D: Organ complications of infants with hypoxic-ischemic encephalopathy before. Ther Hypothermia Temp Manag 11: 58-63, 2021.

12. Munteanu AI, Manea AM, Jinca CM and Boia M: Basic biochemical and hematological parameters in perinatal asphyxia and their correlation with hypoxic ischemic encephalopathy. Exp Ther Med 21: 259, 2021.

13. Hansen AR and Soul JS: Perinatal asphyxia and hypoxic-ischemic encephalopathy. Cloherty and Stark's Manual of Neonatal Care. 8th edition 55: 790-810, 2017.
14. Glass HC and Shellhass RA: Acute symptomatic seizures in neonates. Semin Pediatr Neurol 32: 100768, 2019.

15. Sansevere AJ and Bergin AM: Neonatal seizures in Cloherty and Stark's Manual of Neonatal Care, 8th edition 56: 812-828, 2017.

16. Reddy S, Dutta S and Narang A: Evaluation of lactate dehydrogenase, creatine kinase and hepatic enzymes for the retrospective diagnosis of perinatal asphyxia among sick neonates. Indian Pediatr 45: 144-147, 2008.

17. Alkholy UM, Abdalmonem N, Zaki A, Ali YF, Mohamed SA, Abdelsalam NI, Hashim MIA, Sekkien MA and Elsherbiny YM: Early predictors of brain damage in full-term newborns with hypoxic ischemic encephalopathy. Neuropsychiatr Dis Treat 13: 2133-2139, 2017.

18. Hellstrom-Westas L, Rosen I, de Vries LS and Greisen G: Amplitude-integrated EEG classification and interpretation in preterm and term infants. Neoreviews 7: e76-e87, 2006.

19. Toet MC, Hellström-Westas L, Groenendaal F, Eken P and de Vries LS: Amplitude integrated EEG 3 and $6 \mathrm{~h}$ after birth in full term neonates with hypoxic-ischaemic encephalopathy. Arch Dis Child Fetal Neonatal Ed 81: F19-F23, 1999.

20. Chang PD, Chow DS, Alber A, Lin YK and Youn YA: Predictive values of location and volumetric MRI injury patterns for neurodevelopmental outcomes in hypoxic-ischemic encephalopathy neonates. Brain Sci 10: 1-9, 2020.

21. Hayakawa M, Ito Y, Saito S, Mitsuda N, Hosono S, Yoda H, Cho K, Otsuki K, Ibara S, Terui K, et al: Incidence and prediction of outcome in hypoxic-ischemic encephalopathy in Japan. Pediatr Int 56: 215-221, 2014.

22. Kharoshankaya L, Stevenson NJ, Livingstone V, Murray DM, Murphy BP, Ahearne CE and Boylan GB: Seizure burden and neurodevelopmental outcome in neonates with hypoxic-ischemic encephalopathy. Dev Med Child Neurol 58: 1242-1248, 2016.

23. Beken S, Aydin B, Dilli D, Erol S, Zenciroğlu A and Okumuş N: Can biochemical markers predict the severity of hypoxic-ischemic encephalopathy? Turk Pediatr 56: 62-68, 2014.

24. Hadzimuratovic E, Skrablin S, Hadzimuratovic A and Dinarevic SM: Postasphyxial renal injury in newborns as a prognostic factor of neurological outcome. J Matern Fetal Neonatal Med 27: 407-410, 2014.

25. Sabir H and Hoehn T: Comparison of two amplitude-integrated electroencephalography (aEEG) monitors in term neonates. J Med Syst 41: 114, 2017.

26. Shalak LF, Laptook AR, Velaphi SC and Perlman JM: Amplitudeintegrated electroencephalography coupled with an early neurologic examination enhances prediction of term infants at risk for persistent encephalopathy. Pediatrics 111: 351-357, 2003.

27. Tao JD and Mathur AM: Using amplitude-integrated EEG in neonatal intensive care. J Perinatol 30 (Suppl): S73-S81, 2010.

28. Hellström-Westas L and Rosen I: Continuous brain-function monitoring: State of the art in clinical practice. Semin Fetal Neonat Med 11: 503-511, 2006.

29. Thoresen M, Hellstrom-Westas L, Liu X and de Vries LS: Effect of hypothermia on amplitude-integrated electroencephalogram in infants with asphyxia. Pediatrics 126: e131-e139, 2010.

30. Jose A, Matthai J and Paul S: Correlation of EEG, CT and MRI brain with neurological outcome at 12 months in term newborns with hypoxic ischemic encephalopathy. J Clin Neonatol 2: 125-130, 2013.

31. Glass HC, Glidden D, Jeremy RJ, Barkovich AJ, Ferriero DM and Miller SP: Clinical neonatal seizures are independently associated with outcome in infants at risk for hypoxic-ischemic brain injury. J Pediatr 155: 318-323, 2009.

32. Shankaran S: Neonatal encephalopathy: Treatment with hypothermia. Neo Rev 11: e85-e92, 2010.

33. Hellström-Westas L, Rosén I and Svenningsen NW: Predictive value of early continuous amplitude integrated EEG recordings on outcome after severe birth asphyxia in full term infants. Arch Dis Child Fetal Neonatal 72: F34-F38, 1995.

34. Stamatin M and Paduraru L: Specific medical and ethical aspects in the care of life-threatening illnesses in newborn. Rev Rom Bioet 7: 67-73, 2009.

35. ter Horst HJ, Sommer C, Bergman KA, Fock JM, van Weerden TW and Bos AF: Prognostic significance of amplitude-integrated EEG during the first $72 \mathrm{~h}$ after birth in severely asphyxiated neonates. Pediatr Res 55: 1026-1033, 2004.

36. van Rooij LG, Toet MC, Osredkar D, van Huffelen AC, Groenendaal F and de Vries LS: Recovery of amplitude integrated electroencephalographic background patterns within $24 \mathrm{~h}$ of perinatal asphyxia. Arch Dis Child Fetal Neonatal 90: F245-F251, 2005. 
37. Basti C, Maranella E, Cimini N, Catalucci A, Ciccarelli S, Del Torto M, Di Luca L, Di Natale C, Mareri A, Nardi V, et al: Seizure burden and neurodevelopmental outcome in newborns with hypoxic-ischemic encephalopathy treated with therapeutic hypothermia: A single center observational study. Seizure 83: 154-159, 2020.

38. Mizrahi EM: Neonatal seizures and neonatal epileptic syndromes. Neurol Clin 19: 427-63, 2001.

39. Murray DM, Boylan GB, Ali I, Ryan CA, Murphy BP and Connolly S: Defining the gap between electrographic seizure burden, clinical expression and staff recognition of neonatal seizures. Arch Dis Child Fetal Neonatal Ed 93: F187-F191, 2008.

40. Clancy ARR and Lewis D: Occult neonatal seizures. Epilepsia 29 256-261, 1988.

41. Ronen GM, Buckley D, Penney S and Streiner DL: Long-term prognosis in children with neonatal seizures: A population-based study. Neurology 69: 1816-1822, 2007.

42. Rutherford MA, Pennock JM and Dubowitz LMS: Cranial ultrasound and magnetic resonance imaging in hypoxic ischaemic encephalopathy: A comparison with outcome. Dev Med Child Neurol 36: 813-825, 1994.

43. Nelson K and Ellenberg J: Apgar scores as predictors of chronic neurologic disability. Pediatrics 68: 36-44, 1981

44. Matyas $M$ and Zaharie G: Antioxidants at newborns, antioxidants. Intech Open, 2019. DOI: 10.5772/intechopen.85175. https://www.intechopen.com/books/antioxidants/antioxidants-atnewborns. Accessed May 19, 2019.

45. Perlman JM, Wyllie J, Kattwinkel J, Wyckoff MH, Aziz K, Guinsburg R, Kim HS, Liley HG, Mildenhall L, Simon WM, et al: Part 7: Neonatal resuscitation: 2015 International consensus on cardiopulmonary resuscitation and emergency cardiovascular care science with treatment recommendations. Pediatrics 136 (Suppl 2): S120-S166, 2015.

46. Shoji $\mathrm{H}$ and Koletzko B: Oxidative stress and antioxidant protection in the perinatal period. Curr Opin Clin Nutr Metab Care 10: 324-328, 2007.
47. Laptook AR, Shankaran S, Ambalavanan N, Carlo WA, McDonald SA, Higgins RD and Das A; Hypothermia Subcommittee of the NICHD Neonatal Research Network: Outcome of term infants using apgar scores at $10 \mathrm{~min}$ following hypoxic-ischemic encephalopathy. Pediatrics 124: 1619-1626, 2009.

48. Hankins GDV, Koen S, Gei AF, Lopez SM, Van Hook JW and Anderson GD: Neonatal organ system injury in acute birth asphyxia sufficient to result in neonatal encephalopathy. Obstet Gynecol 99: 688-691, 2002

49. Halvorsen CP, Olson L, Araújo AC, Karlsson M, Nguyễn TT, Khu DTK, Le HTT, Nguyễn HTB, Winbladh B and Russom A: A rapid smartphone-based lactate dehydrogenase test for neonatal diagnostics at the point of care. Sci Rep 9: 9301, 2019.

50. Lv H, Wang Q, Wu S, Yang L, Ren P, Yang Y, Gao J and Li L: Neonatal hypoxic ischemic encephalopathy-related biomarkers in serum and cerebrospinal fluid. Clin Chim Acta 450: 282-297, 2015.

51. Alsina M, Martín-Ancel A, Alarcon-Allen A, Arca G, Gayá F and García-Alix A: The severity of hypoxic-ischemic encephalopathy correlates with multiple organ dysfunction in the hypothermia era. Pediatr Crit Care Med 18: 234-240, 2017.

52. Cristea C, Rotaru O, Burnei-Russu A, Vladareanu R and Vladareanu S: The assessment of fetal neurobehavior by KANET test. Prenatal diagnosis. Editorial Group: MEDICHUB MEDIA. DOI: 10.26416/Peri.2.2.2018.1812, 2018. https://www. medichub.ro/reviste/perinatologia/evaluarea-comportamentuluineurologic-fetal-prin-testul-kanet-id-1812-cmsid-72. Accessed June 23,2018. 